\title{
A high quality composite axion
}

\author{
Benjamin Lillard and Tim M.P. Tait \\ Department of Physics and Astronomy, University of California, \\ Fredrick Reines Hall, Irvine, CA 92697, U.S.A. \\ E-mail: blillard@uci.edu, ttait@uci.edu
}

ABSTRACT: The strong CP problem is a compelling motivation for physics beyond the

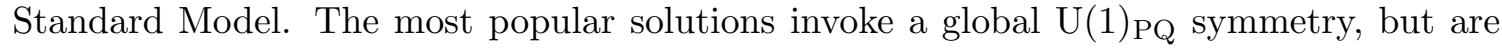
challenged by quantum gravitational corrections which are thought to be incompatible with global symmetries, arguing that realistic theories contain additional structure. We explore a construction in which the $\mathrm{U}(1)_{\mathrm{PQ}}$ symmetry is protected to arbitrary order by virtue of a supersymmetric, confining $\mathrm{SU}(N)_{L} \times \mathrm{SU}(N) \times \mathrm{SU}(N)_{R} \times \mathrm{U}(1)_{X}$ product gauge group, achieving $|\bar{\theta}|<10^{-11}$ for an SU(5) model with $f_{a} \lesssim 3 \times 10^{11} \mathrm{GeV}$. This construction leads to low energy predictions such as a $\mathrm{U}(1)_{X}$ gauge symmetry, and for $X=B-L$ engineers a naturally $\mathcal{O}(\mathrm{TeV})$ value for the $\mu$ parameter of the MSSM.

Keywords: Confinement, Global Symmetries, Spontaneous Symmetry Breaking, Supersymmetric Gauge Theory

ARXIV EPRINT: 1811.03089 


\section{Contents}

1 Introduction 1

1.1 The strong CP problem 1

1.2 Axion quality problem 3

2 Composite axion model $\quad 4$

2.1 Axion quality 6

$2.2 \mathrm{U}(1)_{B-L}$ as $\mathrm{U}(1)_{X}$

2.2.1 Composite Higgs doublets 10

$\begin{array}{lll}2.2 .2 & \text { Color-triplet mesons } & 11\end{array}$

2.3 Alternatives to $B-L \quad 11$

$\begin{array}{lll}2.3 .1 & 5 /-3 / 1 \text { model } & 12\end{array}$

2.3.2 $\mathbf{q}=0$ : neutral MSSM 13

$\begin{array}{lll}2.3 .3 & L_{i}-L_{j} \text { models } & 13\end{array}$

3 Gauge-mediated supersymmetry breaking 13

4 Conclusions and outlook $\quad 15$

\section{Introduction}

Despite the conceptual simplicity of the axion solution to the strong CP problem, relatively few axion models have been developed which naturally predict $|\bar{\theta}| \lesssim 10^{-11}$ when confronted with gravitationally induced $\mathrm{U}(1)_{\mathrm{PQ}}$ violating operators. Models which do sufficiently protect the axion scalar potential from gravitational perturbations typically require large groups or complicated structures, leading to an ongoing search for more satisfying solutions.

In this work we present a relatively simple composite axion model in a confining supersymmetric theory, which is consistent with gauge coupling unification and compatible with current experimental results. Certain mesons in the theory are identified as composite Higgs fields, ameliorating the $B / \mu$ problem of the MSSM, and in one variant of our model the $B-L$ global symmetry of the Standard Model is gauged.

\subsection{The strong CP problem}

The Standard Model (SM) contains several puzzles, one of the most pressing of which is the value of the $\theta$ parameter in the QCD Lagrangian:

$$
\mathcal{L}=\frac{g^{2} \theta}{64 \pi^{2}} \epsilon^{\mu \nu \rho \sigma} G_{\mu \nu}^{a} G_{\rho \sigma}^{a} \equiv \frac{g^{2}}{32 \pi^{2}} \theta G_{\mu \nu} \tilde{G}^{\mu \nu}
$$


Searches for an electric dipole moment of the neutron have so far resulted only in upper limits on its magnitude, implying that $|\bar{\theta}|<6 \times 10^{-11}[1,2]$, where $\bar{\theta}$ is the physically relevant combination of $C P$ violating phases,

$$
\bar{\theta}=\theta+\arg \operatorname{det} M_{Q},
$$

where $M_{Q}$ is the quark mass matrix. As the $\theta$ term violates both $P$ and $C P$, the unnaturally small value of $\bar{\theta}$ is referred to as the strong $C P$ problem. For more complete reviews, see for example [3-5].

In many popular solutions of the strong $C P$ problem, $\bar{\theta}$ is rendered unphysical by ensuring that the classical Lagrangian respects a global $U(1)$ symmetry, which is explicitly broken by the QCD anomaly. A simple example can be seen from eq. (1.2), if one sets $m_{u}=0$ : an axial $\mathrm{U}(1)_{A}$ symmetry emerges in this limit, so that $\arg \operatorname{det} M_{Q}$ (and therefore $\bar{\theta})$ becomes unphysical. If it were not for compelling evidence that $m_{u, d} \neq 0$, this "massless up quark solution" would naturally explain the absence of $C P$ violation in the strong sector.

Axion models address the strong $C P$ problem by associating $\bar{\theta}$ with the pseudo-NambuGoldstone boson of an approximate $\mathrm{U}(1)_{\mathrm{PQ}}$ global symmetry. This is achieved by introducing a (SM singlet) complex scalar $\phi$ together with left-handed color (anti)-triplet fermions $Q$ and $\bar{Q}$, along with the interaction

$$
\mathcal{L} \supset V(\phi)+\phi Q \bar{Q}+\text { h.c. }
$$

where $V(\phi)$ is designed such that $\phi$ acquires an expectation value $\langle\phi\rangle \gtrsim 10^{9} \mathrm{GeV}$. The bare mass term $m Q \bar{Q}$ is forbidden, so that $\mathcal{L}$ respects a $\mathrm{U}(1)_{\mathrm{PQ}}$ symmetry under which $\phi$ is charged. The $\mathrm{SU}(3)_{c}^{2}-\mathrm{U}(1)_{\mathrm{PQ}}$ anomaly coefficient is nonzero, as can be seen from the fact that $(Q \bar{Q})$ carries a net $\mathrm{U}(1)_{\mathrm{PQ}}$ charge.

Expanding about the $\langle\phi\rangle \neq 0$ vacuum, the axion $a$ is identified as the phase of $\phi$ :

$$
\phi=\left(\langle\phi\rangle+\frac{\sigma}{\sqrt{2}}\right) \exp \left(i \frac{a}{f_{a}}\right),
$$

where $f_{a} \equiv \sqrt{2}\langle\phi\rangle$. The $\mathrm{SU}(3)_{c}^{2}-\mathrm{U}(1)_{\mathrm{PQ}}$ anomaly induces an $a G \tilde{G}$ coupling,

$$
\mathcal{L}=\frac{g^{2}}{32 \pi^{2}}\left(\bar{\theta}-\frac{a}{f_{a}}\right) G_{\mu \nu} \tilde{G}^{\mu \nu}
$$

and nonperturbative QCD dynamics generate a periodic potential for a which can be heuristically (up to chiral symmetry-violating corrections [6], which are unimportant for our discussion) described by

$$
V(a) \simeq m_{\pi}^{2} f_{\pi}^{2}\left(1-\cos \left[\frac{a}{f_{a}}-\bar{\theta}\right]\right) .
$$

The axion potential is minimized by $\langle a\rangle=f_{a} \bar{\theta}$, so that $C P$ is conserved in the QCD vacuum.

In "invisible axion" models of this type $[4,7-9]$ the axion is light and weakly coupled, with a mass given by:

$$
m_{a}^{2} \simeq \frac{m_{\pi}^{2} f_{\pi}^{2}}{f_{a}^{2}} .
$$


A lower bound $f_{a} \gtrsim 10^{9} \mathrm{GeV}$ is set primarily by astrophysical observations of stellar cooling and supernovae. In much of the parameter space, the axion provides a natural dark matter candidate: its interactions are suppressed by the decay constant $f_{a}$, and it can be produced in the early universe by the misalignment mechanism [10-12]. For $\mathcal{O}(1)$ initial misalignment angles, the correct relic abundance is obtained for $f_{a} \lesssim 10^{12} \mathrm{GeV}$, though $f_{a}$ could be larger if the misalignment was smaller. The fact that the QCD axion could also play the role of dark matter is one of the reasons for its continued popularity as a solution to the strong $C P$ problem.

\subsection{Axion quality problem}

A closer inspection of the simple axion model presented above reveals a new set of theoretical difficulties, namely a hierarchy problem and a fine-tuning problem. The axion model prefers a hierarchy between the scale of symmetry breaking $f_{a}$ and the Planck mass, $M_{\mathrm{P}}$. A number of standard solutions, such as supersymmetry or compositeness, have been proposed which would render an axion scale $f_{a} \ll M_{\mathrm{P}}$ technically natural. However, many axion models still suffer from a more severe fine-tuning, known as the axion quality problem.

Arguments from general relativity [13-18] suggest that non-perturbative quantum gravitational effects do not respect global symmetries such as baryon number or $\mathrm{U}(1)_{\mathrm{PQ}}$. This is highly problematic for most axion models, which rely on $\mathrm{U}(1)_{\mathrm{PQ}}$ being an exact symmetry in the $\alpha_{s} \rightarrow 0$ limit, explicitly broken only by the QCD anomaly. If additional PQ-violating operators representing the short distance influence of quantum gravity such as

$$
\Delta V(\phi)=\frac{|\phi|^{k+3}}{M_{\mathrm{P}}^{k}}\left(\lambda_{k} \phi+\lambda_{k}^{\star} \phi^{\star}\right)
$$

are present, the corresponding perturbation in $V(a)$ can shift $\langle a\rangle$ far away from the $C P$ conserving value of eq. (1.6):

$$
\delta V(a) \sim \lambda_{k} f_{a}^{4}\left(\frac{f_{a}}{M_{\mathrm{P}}}\right)^{k} \cos \left(\Delta_{\mathrm{PQ}} \frac{a}{f_{a}}-\varphi\right),
$$

where the phase $\varphi$ is determined by $\lambda_{k}$, and $\Delta_{\mathrm{PQ}}$ is the $\mathrm{U}(1)_{\mathrm{PQ}}$ charge of the operator $\phi$. It is convenient to describe such perturbations by defining a "quality factor" $Q$ :

$$
\delta V(a)=Q f_{a}^{4} \cos \left(\frac{a}{f_{a}}-\varphi\right) .
$$

If we assume $\varphi \sim \mathcal{O}(1)$ is not tuned, the measured value of $|\bar{\theta}| \lesssim 10^{-11}$ is possible only if $\delta V(a)$ satisfies

$$
Q \lesssim 10^{-63}\left(\frac{10^{12} \mathrm{GeV}}{f_{a}}\right)^{4}
$$

Satisfying this bound requires that the theory of quantum gravity somehow produce a severe fine-tuning in the $\lambda_{k}$, such that even the dimension-12 operators in eq. (1.9) must have $\lambda_{k} \ll 1$.

In a truly compelling axion model, the $\mathrm{U}(1)_{\mathrm{PQ}}$ symmetry should emerge as a consequence of some other underlying structure which forbids the problematic operators. For 
example, a gauged discrete $\mathbb{Z}_{n}$ symmetry [19] for some $n \gtrsim 13$ can forbid all PQ-violating operators smaller than $\left(\phi^{n}+\right.$ c.c. $)$. Significantly smaller groups can be employed to the same effect in supersymmetric theories [20,21], if the discrete group is an $R$ symmetry. Composite axion models such as [22-24] also protect $\mathrm{U}(1)_{\mathrm{PQ}}$ to arbitrarily high order, with the added benefit that the axion scale $f_{a}$ can be generated dynamically. Other constructions [25-27] associate $\mathrm{U}(1)_{\mathrm{PQ}}$ with a different, gauged $\mathrm{U}(1)$, so that many of the PQ-violating operators are forbidden. Many of these constructions are intricate and also rather delicate in the sense that the axion quality is easily ruined in extensions of the model.

In this work we present an alternative composite axion model based on an $\mathrm{SU}(N) \times$ $\mathrm{SU}(N)$ confining supersymmetric gauge theory with simple matter content. The Standard Model matter fields and interactions are easily embedded, and we show that the axion quality is preserved even with the addition of new fields. Upon identifying the $H_{u}$ and $H_{d}$ doublets as mesons from $\mathrm{SU}(N)$ confinement, we find that the $\mu$ parameter of the MSSM naturally assumes an $\mathcal{O}(\mathrm{TeV})$ value. Finally, we explore the ability of this model to mediate supersymmetry breaking via composite messengers.

\section{Composite axion model}

Conjectured dualities [28, 29] allow one to analyze the low energy behavior of supersymmetric gauge theories. In particular, an $\mathrm{SU}\left(N_{c}\right)$ gauge theory with $N_{f}=N_{c}$ flavors of quarks $(Q+\bar{Q})$ in the (anti-)fundamental representation is expected to confine at a characteristic scale $\Lambda$, such that the low energy degrees of freedom are described by the gauge-singlet operators

$$
M=(Q \bar{Q}), \quad B=\left(Q^{N}\right), \quad \bar{B}=\left(\bar{Q}^{N}\right),
$$

subject to the quantum-modified constraint

$$
\operatorname{det} M-B \bar{B}=\Lambda^{2 N}
$$

The constraint eq. (2.2) guarantees that the global $\mathrm{SU}\left(N_{f}\right) \times \mathrm{SU}\left(N_{f}\right) \times \mathrm{U}(1)$ symmetry is spontaneously broken, either by $\langle M\rangle \neq 0$ or $\langle B \bar{B}\rangle \neq 0$. Similar behavior has been demonstrated in theories with product gauge groups of the form $\mathrm{SU}(N) \times \mathrm{SU}(N) \times \ldots \times$ $\mathrm{SU}(N)$ with bifundamental matter [30]. We show that a composite axion emerges in a subset of these theories, with sufficiently high axion quality.

We invoke the gauge group $\mathrm{SU}(N)_{L} \times \mathrm{SU}(N)_{S M} \times \mathrm{SU}(N)_{R} \times \mathrm{U}(1)_{X}$, where $\mathrm{SU}(N)_{S M}$ contains the Standard Model $\mathrm{SU}(3)_{c} \times \mathrm{SU}(2)_{L} \times \mathrm{U}(1)_{Y}$ either as a gauged subgroup or as an $\mathrm{SU}(5)$ grand unified theory. The strongly coupled $\mathrm{SU}(N)_{L, R}$ confine at the characteristic scales $\Lambda_{L, R} \gg \mathrm{TeV}$, but the Abelian $\mathrm{U}(1)_{X}$ is weakly coupled. ${ }^{1}$ The bifundamental fields $\bar{Q}_{1,2}$ and $Q_{1,2}$ have $\mathrm{U}(1)_{X}$ charges \pm 1 , as depicted in the moose diagram of figure 1 , with $\mathrm{U}(1)_{\mathrm{PQ}}$ charges shown in table 1 .

\footnotetext{
${ }^{1}$ The axion construction leaves the charges of the MSSM matter under $\mathrm{U}(1)_{X}$ largely undetermined. We explore several alternatives below.
} 




Figure 1. Moose diagram indicating the charges of bifundamental matter fields $\bar{Q}_{1,2}$ and $Q_{1,2}$ under the gauge group $\mathrm{SU}(N)_{L} \times \mathrm{SU}(N)_{S M} \times \mathrm{SU}(N)_{R} \times \mathrm{U}(1)_{X}$ and global $\mathrm{SU}(N)_{1} \times \mathrm{SU}(N)_{2}$ global symmetries. The Standard Model SU $(3)_{c} \times \mathrm{SU}(2)_{L} \times \mathrm{U}(1)_{Y}$ is a subgroup of $G_{0}$.

\begin{tabular}{|c|c|ccc|c|c|c|}
\hline & $\mathrm{SU}(N)_{1}$ & $G_{L}$ & $G_{0}$ & $G_{R}$ & $\mathrm{SU}(N)_{2}$ & $\mathrm{U}(1)_{X}$ & $\mathrm{U}(1)_{\mathrm{PQ}}$ \\
\hline $\bar{Q}_{2}$ & $\square$ & $\square$ & & & & 1 & $-(1-\alpha) / N$ \\
$\bar{Q}_{1}$ & & $\square$ & $\square$ & & & -1 & $(1-\alpha) / N$ \\
$Q_{1}$ & & & $\square$ & $\bar{\square}$ & & 1 & $(1+\alpha) / N$ \\
$Q_{2}$ & & & & $\square$ & $\square$ & -1 & $-(1+\alpha) / N$ \\
\hline $\bar{M}$ & $\square$ & & $\square$ & & & 0 & 0 \\
$M$ & & & $\square$ & & $\square$ & 0 & 0 \\
$\bar{B}_{2}$ & & & & & & $N$ & $-1+\alpha$ \\
$\bar{B}_{1}$ & & & & & & $-N$ & $1-\alpha$ \\
$B_{1}$ & & & & & & $N$ & $1+\alpha$ \\
$B_{2}$ & & & & & & $-N$ & $-1-\alpha$ \\
\hline
\end{tabular}

Table 1. U(1) $)_{\mathrm{PQ}}$ charges and representations under the gauged $G_{L} \times G_{0} \times G_{R}$ and the global $\mathrm{SU}(N)_{1} \times \mathrm{SU}(N)_{2}$ symmetries are indicated for the bifundamental quarks (upper half) and composite operators resulting from $G_{L} \times G_{R}$ confinement (lower half).

Below the scales $\Lambda_{L}$ and $\Lambda_{R}$, the low energy degrees of freedom are described by the composite operators satisfying equations of motion:

$$
\begin{aligned}
& \bar{M}=\left(\bar{Q}_{2} \bar{Q}_{1}\right) \quad \bar{B}_{1}=\left(\bar{Q}_{1}^{N}\right) \quad \bar{B}_{2}=\left(\bar{Q}_{2}^{N}\right) \quad \Lambda_{L}^{2 N}=\operatorname{det} \bar{M}-\bar{B}_{1} \bar{B}_{2} \\
& M=\left(Q_{1} Q_{2}\right) \quad B_{1}=\left(Q_{1}^{N}\right) \quad B_{2}=\left(Q_{2}^{N}\right) \quad \Lambda_{R}^{2 N}=\operatorname{det} M-B_{1} B_{2} .
\end{aligned}
$$

In the absence of a superpotential, this model respects the global $\mathrm{SU}(N)_{1} \times \mathrm{SU}(N)_{2}$ symmetries shown in figure 1 , as well the gauged $\mathrm{U}(1)_{X}$. There is also a conserved $\mathrm{U}(1)_{R}$, under which the gauginos have charge +1 and all of the $\bar{Q}_{1,2}$ and $Q_{1,2}$ are neutral, which remains unbroken everywhere on the moduli space.

In the regime where $G_{0}$ is weakly coupled, there is another nearly exact global symmetry, $\mathrm{U}(1)_{\mathrm{PQ}}$, which is broken only by the $G_{0}^{2} \mathrm{U}(1)_{\mathrm{PQ}}$ anomaly. Due to the locally conserved $\mathrm{U}(1)_{X}$, there is no unique assignment of Peccei-Quinn charges: rotations under $\mathrm{U}(1)_{\mathrm{PQ}}$ can always be combined with a global $\mathrm{U}(1)_{X}$ transformation to define a new, equally valid Peccei-Quinn symmetry. This degeneracy is parameterized by the parameter $\alpha$ in table 1 .

On the quantum-deformed moduli space described by eq. (2.3), the global $\mathrm{SU}(N)_{1} \times$ $\mathrm{SU}(N)_{2} \times \mathrm{U}(1)_{X} \times \mathrm{U}(1)_{\mathrm{PQ}}$ symmetry must be broken to a subgroup. Furthermore, if the 
low energy limit of this theory is to approach the Standard Model, then it must be true that $\operatorname{det} M=\operatorname{det} \bar{M}=0$; otherwise, $\mathrm{SU}(3)_{c}$ would be broken in the vacuum. The vacuum therefore must be engineered to lie on the $\left\langle B_{1} B_{2}\right\rangle \neq 0,\left\langle\bar{B}_{1} \bar{B}_{2}\right\rangle \neq 0$ branch of the moduli space, where $\mathrm{U}(1)_{X}$ and $\mathrm{U}(1)_{\mathrm{PQ}}$ are both spontaneously broken, and the $\mathrm{U}(1)_{X}$ vector supermultiplet acquires a mass by "eating" a combination of the chiral superfields. This is accomplished by including a term in the superpotential of the form:

$$
\frac{\left(\bar{Q}_{2} \bar{Q}_{1}\right)\left(Q_{1} Q_{2}\right)}{M_{*}}
$$

which after confinement generates a mass term for the mesons, $W \sim \mu \bar{M} M$, lifting the mesonic flat directions. If not otherwise present, this term is expected to be induced by quantum gravitational effects.

A unique definition of the Peccei-Quinn charges emerges once $\mathrm{U}(1)_{X}$ is broken: by canonically normalizing the kinetic terms of the (would-be) Nambu-Goldstone bosons of $\mathrm{U}(1)_{\mathrm{PQ}}$ and $\mathrm{U}(1)_{X}$, the parameter $\alpha$ of table 1 is related to the vacuum expectation values (VEVs) of the baryons as

$$
\alpha=\frac{\bar{v}_{1}^{2}+\bar{v}_{2}^{2}-v_{1}^{2}-v_{2}^{2}}{f_{X}^{2}}
$$

where

$$
\bar{v}_{i}^{2}=2\left|\frac{\left\langle\bar{B}_{i}\right\rangle}{\Lambda_{L}^{N-1}}\right|^{2}, \quad v_{i}^{2}=2\left|\frac{\left\langle B_{i}\right\rangle}{\Lambda_{R}^{N-1}}\right|^{2}, \quad f_{X}^{2}=\bar{v}_{1}^{2}+\bar{v}_{2}^{2}+v_{1}^{2}+v_{2}^{2},
$$

and where the axion decay constant $f_{a}$ is

$$
f_{a}^{2}=f_{X}^{2}\left(1-\alpha^{2}\right)
$$

With this normalization, a $\mathrm{U}(1)_{\mathrm{PQ}}$ rotation by a phase $\theta$ is achieved by the linear shift

$$
a \rightarrow a+\theta f_{a}
$$

Although the products $v_{1} v_{2}$ and $\bar{v}_{1} \bar{v}_{2}$ are set by the quantum modified constraints,

$$
\bar{v}_{1} \bar{v}_{2}=2\left|\Lambda_{L}^{2}\right|, \quad v_{1} v_{2}=2\left|\Lambda_{R}^{2}\right|
$$

the values of the decay constants $f_{a}$ and $f_{X}$ vary along the flat directions within the allowed ranges

$$
f_{X}^{2} \geq 4\left|\Lambda_{L}^{2}\right|+4\left|\Lambda_{R}^{2}\right|, \quad f_{a}^{2} \leq f_{X}^{2} .
$$

The case $f_{a} \ll f_{X}$ is achieved in the limits $\Lambda_{L} \gg \Lambda_{R}$ or $\Lambda_{L} \ll \Lambda_{R}$, as $\alpha \rightarrow \pm 1$. Conversely, the special case $v_{1}^{2}+v_{2}^{2}=\bar{v}_{1}^{2}+\bar{v}_{2}^{2}$ corresponds to $f_{a}=f_{X}$.

\subsection{Axion quality}

To examine the axion quality, we introduce operators characterized by $M_{\mathrm{P}}$ which represent an effective field theory description of the low energy residual effects of quantum gravity. 
It is convenient to introduce a set of rescaled composite operators with mass dimension +1 :

$$
\overline{\mathcal{M}}=\frac{\left(\bar{Q}_{2} \bar{Q}_{1}\right)}{\Lambda_{L}} \quad \mathcal{M}=\frac{\left(Q_{1} Q_{2}\right)}{\Lambda_{R}} \quad \overline{\mathcal{B}}_{i}=\frac{\left(\bar{Q}_{i}^{N}\right)}{\Lambda_{L}^{N-1}} \quad \mathcal{B}_{i}=\frac{\left(Q_{i}^{N}\right)}{\Lambda_{R}^{N-1}}
$$

The effective gravitational superpotential violating all of the global symmetries takes the form:

$$
\begin{aligned}
W_{g} & =\lambda_{1} \frac{\left(\bar{Q}_{1}^{N}\right)\left(Q_{1}^{N}\right)}{M_{\mathrm{P}}^{2 N-3}}+\lambda_{2} \frac{\left(\bar{Q}_{2}^{N}\right)\left(Q_{2}^{N}\right)}{M_{\mathrm{P}}^{2 N-3}}+\lambda_{3} \frac{\left(\bar{Q}_{2}^{N}\right)\left(\bar{Q}_{1}^{N}\right)}{M_{\mathrm{P}}^{2 N-3}}+\lambda_{4} \frac{\left(Q_{1}^{N}\right)\left(Q_{2}^{N}\right)}{M_{\mathrm{P}}^{2 N-3}}+\rho_{1} \frac{\left(\bar{Q}_{2} \bar{Q}_{1}\right)\left(Q_{1} Q_{2}\right)}{M_{\mathrm{P}}}+\ldots \\
& =\left(\frac{\Lambda_{L}^{N-1} \Lambda_{R}^{N-1}}{M_{\mathrm{P}}^{2 N-3}}\right)\left\{\lambda_{1} \overline{\mathcal{B}}_{1} \mathcal{B}_{1}+\lambda_{2} \overline{\mathcal{B}}_{2} \mathcal{B}_{2}+\lambda_{3} \overline{\mathcal{B}}_{1} \overline{\mathcal{B}}_{2}+\lambda_{4} \mathcal{B}_{1} \mathcal{B}_{2}\right\}+\rho_{1}\left(\frac{\Lambda_{L} \Lambda_{R}}{M_{\mathrm{P}}}\right) \overline{\mathcal{M}} \mathcal{M}+\ldots
\end{aligned}
$$

with parameters $\lambda_{i}$ and $\rho_{i}$ encoding the UV physics. Of the operators listed above, only the two associated with $\lambda_{1}$ and $\lambda_{2}$ violate $\mathrm{U}(1)_{\mathrm{PQ}}$. All of the lower-dimensional operators such as $\left(\bar{Q}_{2} \bar{Q}_{1}\right)\left(Q_{1} Q_{2}\right)$ are neutral under $\mathrm{U}(1)_{\mathrm{PQ}}$, and thus not harmful to the axion quality.

In a supersymmetric vacuum, the leading $\mathrm{U}(1)_{\mathrm{PQ}}$ violation appears with $M_{\mathrm{P}}^{4 N-6}$ suppression in the Lagrangian: for example, within terms such as

$$
\left|\frac{\partial W_{g}}{\partial \mathcal{B}_{1}}\right|^{2}=\left|\frac{\Lambda_{L}^{N-1} \Lambda_{R}^{N-1}}{M_{\mathrm{P}}^{2 N-3}}\right|^{2}\left|\lambda_{1} \overline{\mathcal{B}}_{1}+\lambda_{4} \mathcal{B}_{2}\right|^{2}
$$

implying a perturbation to the axion potential on the order of

$$
Q f_{a}^{4} \sim\left|\lambda_{1} \lambda_{4}\right|\left(\frac{\sqrt{\Lambda_{L} \Lambda_{R}}}{M_{\mathrm{P}}}\right)^{4 N-4} M_{\mathrm{P}}^{2}\left\langle\overline{\mathcal{B}}_{1}\right\rangle\left\langle\mathcal{B}_{2}\right\rangle
$$

Taking $\Lambda_{L} \approx \Lambda_{R} \approx f_{a} \approx 10^{11} \mathrm{GeV}$ as a benchmark and ignoring $\mathcal{O}(1)$ factors, the quality factor

$$
Q \sim\left|\lambda_{1} \lambda_{4}\right| 10^{48-32 N}
$$

satisfies the bound given in eq. (1.11) for $N>3$, even when the $\lambda_{i}$ are $\mathcal{O}(1)$.

More serious perturbations to the axion potential emerge when supersymmetry breaking is taken into account. Supersymmetry breaking induces an " $A$-term" potential,

$$
-\mathcal{L}_{A}=\left(\frac{\Lambda_{L}^{N-1} \Lambda_{R}^{N-1}}{M_{\mathrm{P}}^{2 N-3}}\right)\left\{A_{1} \lambda_{1} \overline{\mathcal{B}}_{1} \mathcal{B}_{1}+A_{2} \lambda_{2} \overline{\mathcal{B}}_{2} \mathcal{B}_{2}+A_{3} \lambda_{3} \overline{\mathcal{B}}_{1} \overline{\mathcal{B}}_{2}+A_{4} \lambda_{4} \mathcal{B}_{1} \mathcal{B}_{2}\right\}+\text { h.c }
$$

where the mass scales $A_{i}$ are in principle calculable once a particular mechanism of supersymmetry breaking is specified. To remain agnostic concerning the details of supersymmetry-breaking, we assume that the $A_{i}$ should be of roughly the same magnitude as the $\mathrm{SU}(3)_{c} \times \mathrm{SU}(2)_{L} \times \mathrm{U}(1)_{Y}$ gaugino masses.

Both the $A_{1}$ and $A_{2}$ terms in eq. (2.17) perturb the axion potential:

$$
\delta V(a)=2 \frac{\Lambda_{L}^{N-1} \Lambda_{R}^{N-1}}{M_{\mathrm{P}}^{2 N-3}}\left\{\left|A_{1} \lambda_{1}\left\langle\overline{\mathcal{B}}_{1}\right\rangle\left\langle\mathcal{B}_{1}\right\rangle\right| \cos \left(2 \frac{a}{f_{a}}+\varphi_{1}\right)+\left|A_{2} \lambda_{2}\left\langle\overline{\mathcal{B}}_{2}\right\rangle\left\langle\mathcal{B}_{2}\right\rangle\right| \cos \left(2 \frac{a}{f_{a}}+\varphi_{2}\right)\right\} .
$$


Again taking $\Lambda_{L, R} \approx f_{a} \approx 10^{11} \mathrm{GeV}$, the constraint on the quality factor eq. (1.11) can be written as

$$
\frac{\lambda_{i} A_{i}}{10^{4} \mathrm{GeV}}\left(\frac{10^{19} \mathrm{GeV}}{M_{\mathrm{P}}}\right)^{2 N-3}\left(\frac{\Lambda_{L} \Lambda_{R}}{10^{22} \mathrm{GeV}^{2}}\right)^{N-1} \frac{\left\langle\overline{\mathcal{B}}_{i}\right\rangle\left\langle\mathcal{B}_{i}\right\rangle}{10^{22} \mathrm{GeV}^{2}} \cdot 10^{-16 N} \lesssim 10^{-76}
$$

for $i=1,2$, indicating that models with $N \geq 5$ are free from fine-tuning as long as the characteristic scales $\Lambda_{L, R}$ and $f_{a}$ are not much larger than $10^{11} \mathrm{GeV}$.

In figure 2 we plot the maximum values of $\lambda_{i}$ consistent with eq. (2.19), for given values of $f_{a}, N$, and the other parameters, with the simplifying assumptions $A_{1} \approx A_{2}$ and $\lambda_{1} \approx \lambda_{2}$. It is convenient to label the vacua with the following parameterization:

$$
\tan \beta_{L}=\frac{\bar{v}_{2}}{\bar{v}_{1}} \quad \tan \beta_{R}=\frac{v_{2}}{v_{1}} \quad \sin ^{2} 2 \gamma=\frac{f_{a}^{2}}{f_{X}^{2}}=1-\alpha^{2} .
$$

All of the dimensionful parameters except for $A_{i}$ and $M_{\mathrm{P}}$ are now expressed in terms of $f_{a}$ :

$$
\bar{v}_{1}=\frac{\cos \beta_{L}}{2 \cos \gamma} f_{a} \quad \bar{v}_{2}=\frac{\sin \beta_{L}}{2 \cos \gamma} f_{a} \quad v_{1}=\frac{\cos \beta_{R}}{2 \sin \gamma} f_{a} \quad v_{2}=\frac{\sin \beta_{R}}{2 \sin \gamma} f_{a},
$$

so that the axion quality condition is expressed:

$$
\begin{aligned}
\frac{Q f_{a}^{4}}{M_{\mathrm{P}}^{4}}= & 8\left(\frac{f_{a}^{2}}{8 M_{\mathrm{P}}^{2} \sin 2 \gamma}\right)^{N}\left(\sin 2 \beta_{L} \sin 2 \beta_{R}\right)^{\frac{N-1}{2}} \\
& \times\left(\frac{\lambda_{1} A_{1} \cos \beta_{L} \cos \beta_{R}+\lambda_{2} A_{2} \sin \beta_{L} \sin \beta_{R}}{M_{\mathrm{P}}}\right) \lesssim 10^{-88} .
\end{aligned}
$$

Because $\beta_{L, R}$ label degenerate vacua on the moduli space defined by eq. (2.3), particularly large or small values of $\tan \beta_{L, R}$ are typically unnatural. On the other hand, $\gamma$ is primarily determined by the ratio $\Lambda_{L} / \Lambda_{R}$ :

$$
\tan \gamma=\frac{\Lambda_{L}}{\Lambda_{R}} \sqrt{\frac{\sin 2 \beta_{L}}{\sin 2 \beta_{R}}},
$$

so large or small values of $\tan \gamma$ are more easily tolerated from a naturalness perspective. As we see from eq. (2.22), the best axion quality is achieved for $\tan \gamma \approx 1$, when $f_{a} \approx f_{X}$ and $\Lambda_{L} \approx \Lambda_{R}$.

We show the maximum tolerable $\lambda_{1} \approx \lambda_{2}$ as a function of $f_{a}$ for a few choices of $N, \tan \beta_{L}=\tan \beta_{R}$, and $\sin 2 \gamma$ in figure 2 . While effective field theory would suggest that generic theories of quantum gravity should produce $\lambda_{1,2} \sim \mathcal{O}(1)$, in [16-18] it is argued that wormhole-induced $\mathrm{U}(1)_{\mathrm{PQ}}$ violation yields suppressed values of $\lambda_{i} \sim \exp \left(-S_{w}\right)$, where the wormhole action $S_{w}$ depends logarithmically on the axion decay constant, $S_{w} \sim a-b \ln \frac{f_{a}}{M_{\mathrm{P}}}$. For typical cases the resulting suppression in $\lambda_{i}$ is modest: values as small as $\lambda \sim 10^{-7}$ are achieved in [16] for $f_{a} \sim 10^{12} \mathrm{GeV}$. For $N=5$ such that $G_{0}$ is large enough to contain the SM, $\mathcal{O}(1) \lambda$ 's are consistent with $f_{a} \lesssim 10^{11} \mathrm{GeV}$.

Generally, the high axion quality observed in eq. (2.19) is preserved even when new fields are coupled to the model provided that they are neutral under $\mathrm{U}(1)_{X}$. Problems arise if there are fields $S$ with $\mathrm{U}(1)_{X}$ charges:

$$
q_{S}= \pm N, \pm \frac{N}{2}, \pm \frac{N}{3}, \ldots, \pm \frac{N}{N-1},
$$

for which case $W_{g}$ includes gauge-invariant terms $S^{p} B_{1,2}$ or $S^{p} \bar{B}_{1,2}$ for some power $p<N$. 


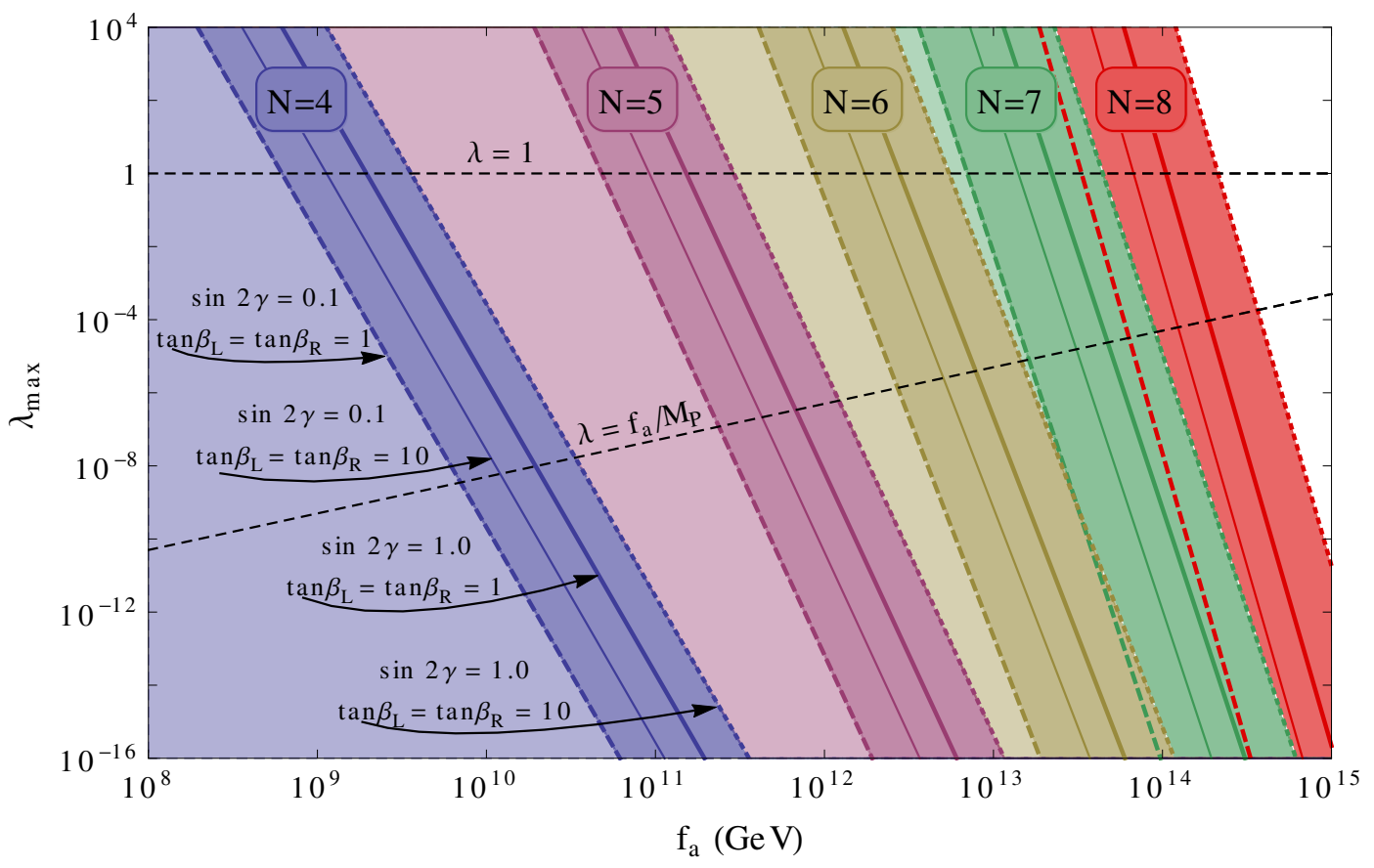

Figure 2. Maximum values of $\lambda_{1} \approx \lambda_{2}$ consistent with eq. (2.22) for given values of $f_{a}$ and $N=4,5,6,7,8$. The region to the left of each line indicates the axion models which return $|\bar{\theta}|<10^{-11}$ without any fine tuning. From left to right within each band of a given $N$, models are indicated with: $\sin 2 \gamma=0.1, \tan \beta_{L}=\tan \beta_{R}=1$ (thin, dashed); $\sin 2 \gamma=0.1, \tan \beta_{L}=\tan \beta_{R}=10$ (thin, solid); $\sin 2 \gamma=\tan \beta_{L}=\tan \beta_{L}=1$ (thick, solid); and $\sin 2 \gamma=1, \tan \beta_{L}=\tan \beta_{L}=10$ (thin, dotted). In each case $A_{1} \approx A_{2}=10^{5} \mathrm{GeV}$.

\section{$2.2 \mathrm{U}(1)_{B-L}$ as $\mathrm{U}(1)_{X}$}

From eq. (2.19) we see the remarkable fact that for $f_{a} \lesssim 10^{11} \mathrm{GeV}$ and $\mathcal{O}(1)$ values in the couplings $\lambda_{i}$, sufficient protection of the axion quality requires $N \geq 5$ : precisely the right size to fit the entire Standard Model within $G_{0}$. In this section we take $G_{0}=\mathrm{SU}(5)$ to be a global symmetry with a gauged $\mathrm{SU}(3)_{c} \times \mathrm{SU}(2)_{L} \times \mathrm{U}(1)_{Y}$ subgroup, and we identify $\mathrm{U}(1)_{X}$ as the $B-L$ symmetry of the Standard Model. The mesons $\mathcal{M}(\mathbf{5})$ and $\overline{\mathcal{M}}(\overline{\mathbf{5}})$ decompose into irreducible representations of $\mathrm{SU}(3) \times \mathrm{SU}(2) \times \mathrm{U}(1)$ :

$$
\begin{aligned}
& \mathcal{M}(\mathbf{5}) \longrightarrow \mathcal{M}^{(3)}(\mathbf{3}, \mathbf{1})_{-\frac{1}{3}} \oplus \mathcal{M}^{(2)}(\mathbf{1}, \mathbf{2})_{\frac{1}{2}} \\
& \overline{\mathcal{M}}(\overline{\mathbf{5}}) \longrightarrow \overline{\mathcal{M}}^{(3)}(\overline{\mathbf{3}}, \mathbf{1})_{\frac{1}{3}} \oplus \overline{\mathcal{M}}^{(2)}(\mathbf{1}, \mathbf{2})_{-\frac{1}{2}} .
\end{aligned}
$$

Table 2 indicates the representations of the composites under the SM, plus three generations of MSSM matter and three right-handed neutrinos necessary to cancel the $\mathrm{U}(1)_{B-L}$ gauge anomaly.

The $B-L$ charges of the baryons $\mathcal{B}_{i}$ and $\overline{\mathcal{B}}_{i}$ are left in terms of a constant $q \neq 0$ which parameterizes their size relative to the canonical charges of the MSSM matter. While generic values of $q$ are phenomenologically viable, certain choices would permit low-dimensional $\mathrm{U}(1)_{\mathrm{PQ}}$-violating operators and spoil the axion quality. The problematic 


\begin{tabular}{|c|c|ccc|c|c|c|}
\hline & $\mathrm{SU}(5)_{1}$ & $S U_{3}$ & $S U_{2}$ & $\mathrm{U}(1)_{Y}$ & $\mathrm{SU}(5)_{2}$ & $\mathrm{U}(1)_{B-L}$ & $\mathrm{U}(1)_{\mathrm{PQ}}$ \\
\hline$\overline{\mathcal{M}}^{(3)}$ & $\mathbf{5}$ & $\overline{\mathbf{3}}$ & & $1 / 3$ & & 0 & 0 \\
$\overline{\mathcal{M}}^{(2)}$ & $\mathbf{5}$ & & $\mathbf{2}$ & $-1 / 2$ & & 0 & 0 \\
\hline $\mathcal{M}^{(3)}$ & & $\mathbf{3}$ & & $-1 / 3$ & $\overline{\mathbf{5}}$ & 0 & 0 \\
$\mathcal{M}^{(2)}$ & & & $\mathbf{2}$ & $1 / 2$ & $\overline{\mathbf{5}}$ & 0 & 0 \\
\hline$Q_{L}$ & & $\mathbf{3}$ & $\mathbf{2}$ & $1 / 6$ & & $+1 / 3$ & 0 \\
$\bar{u}_{R}$ & & $\overline{\mathbf{3}}$ & & $-2 / 3$ & & $-1 / 3$ & 0 \\
$\bar{d}_{R}$ & & $\overline{3}$ & & $1 / 3$ & & $-1 / 3$ & 0 \\
\hline$L$ & & & $\mathbf{2}$ & $-1 / 2$ & & +1 & 0 \\
$\bar{e}_{R}$ & & & & +1 & & -1 & 0 \\
$\bar{\nu}_{R}$ & & & & 0 & & -1 & 0 \\
\hline $\mathcal{B}_{1}, \overline{\mathcal{B}}_{2}$ & & & & 0 & & $5 q$ & $\pm 1+\alpha$ \\
$\overline{\mathcal{B}}_{1}, \mathcal{B}_{2}$ & & & & 0 & & $-5 q$ & $\pm 1-\alpha$ \\
\hline
\end{tabular}

Table 2. Transformation representations of the superfields for the $\mathrm{U}(1)_{X}=\mathrm{U}(1)_{B-L}$ model.

$q$ can be identified by considering all of the low-dimensional SU $(5)_{\text {SM }}$ singlet operators with nonzero $B-L$ charge:

$$
\left(\bar{\nu}_{R}\right)_{-1}, \quad\left(\bar{\nu}_{R}^{n}\right)_{-n}, \quad\left(L \overline{\mathcal{M}}^{(2)}\right)_{+1}, \quad\left(\bar{d}_{R} \mathcal{M}^{(3)}\right)_{-1 / 3}, \quad\left(\overline{\mathcal{M}}^{(3)} Q_{L} L\right)_{+1 / 3},
$$

where the subscripts indicate the $B-L$ charge of each operator. Since none of these carry PQ charge, the superpotential operator constructed by multiplying any of them by a baryon superfield would violate $\mathrm{U}(1)_{\mathrm{PQ}}$ unacceptably. To avoid this issue, we restrict ourselves to the cases where $q \neq \pm \frac{n}{5}$, for $n=0,1,2,3,4$, and also $q \neq \pm \frac{1}{3}$.

\subsubsection{Composite Higgs doublets}

The identification of $X=B-L$ has positive implications for the superpotential, notably by forbidding many of the operators that would mediate highly constrained $B$ and/or $L$ violation such as proton decay [31]. The allowed low energy effective superpotential has the form:

$W=\mu \overline{\mathcal{M}}^{(2)} \mathcal{M}^{(2)}+\mu^{\prime} \overline{\mathcal{M}}^{(3)} \mathcal{M}^{(3)}+y_{u} Q_{L} \mathcal{M}^{(2)} \bar{u}_{R}+y_{d} Q_{L} \overline{\mathcal{M}}^{(2)} \bar{d}_{R}+y_{e} L \overline{\mathcal{M}}^{(2)} \bar{e}_{R}+y_{\nu} L \mathcal{M}^{(2)} \bar{\nu}_{R}$,

containing mass terms for the doublet and triplet mesons, and Yukawa interactions for the doublets with the MSSM matter.

The mesons $\overline{\mathcal{M}}^{(2)}$ and $\mathcal{M}^{(2)}$ have the same gauge representations as the MSSM Higgs superfields $H_{d}$ and $H_{u}$. We take the economical route of interpreting the lightest $\overline{\mathcal{M}}^{(2)}+\mathcal{M}^{(2)}$ pair of the five flavors of $\mathrm{SU}(2)_{L}$ doublet mesons as composite MSSM Higgs superfields, which potentially offers insight into the $\mu$ problem of the MSSM. The terms in eq. (2.28) descend from non-renormalizable composite operators in the UV theory. In the case of the $\mu$ terms, these operators are dimension- 4 and violate the $\mathrm{U}(1)_{R}$ 
symmetry. If generated by quantum gravitational residuals, the natural mass scale for $\mu$ and $\mu^{\prime}$ would thus be:

$$
W_{g} \sim \frac{\left(\bar{Q}_{2} \bar{Q}_{1}\right)\left(Q_{1} Q_{2}\right)}{M_{\mathrm{P}}} \longrightarrow \frac{\Lambda_{L} \Lambda_{R}}{M_{\mathrm{P}}}\left(\overline{\mathcal{M}}^{(2)} \mathcal{M}^{(2)}+\overline{\mathcal{M}}^{(3)} \mathcal{M}^{(3)}\right) \longrightarrow \mu, \mu^{\prime} \sim \frac{\Lambda_{L} \Lambda_{R}}{M_{\mathrm{P}}}
$$

This is $\mu \sim \mathcal{O}(\mathrm{TeV})$ for our benchmark choice of $\Lambda_{L} \approx \Lambda_{R} \approx 10^{11} \mathrm{GeV}$.

The Yukawa interactions of eq. (2.28) similarly correspond to dimension five operators in the UV. Realizing the large couplings necessary for the heavy quarks requires that they be generated at a lower scale $M_{F} \ll M_{\mathrm{P}}$ :

$$
W=y_{u}^{\prime} \frac{Q_{L}\left(Q_{1} Q_{2}\right) \bar{u}_{R}}{M_{F}}+y_{d}^{\prime} \frac{Q_{L}\left(\bar{Q}_{2} \bar{Q}_{1}\right) \bar{d}_{R}}{M_{F}}+y_{e}^{\prime} \frac{L\left(\bar{Q}_{2} \bar{Q}_{1}\right) \bar{e}_{R}}{M_{F}}
$$

where $y_{t} \sim 1$ requires $M_{F} \sim \Lambda_{R}$ (and $y_{b}$ requires $\Lambda_{L}$ is not much larger). Unlike the dynamics generating the $\mu$ terms, the Yukawa interactions are compatible with the $\mathrm{U}(1)_{R}$ symmetry, which allows for the disparate scales to remain technically natural.

The presence of the four additional $\mathcal{M}^{(2)}$ and $\overline{\mathcal{M}}^{(2)}$ in eq. (2.30) poses a potential phenomenological problem. In the absence of any additional structure, the $y_{u, d, e}^{\prime}$ couplings of the matter fields with the heavier $\mathrm{SU}(2)_{L}$ doublets will generally introduce flavor-changing neutral currents (FCNC). A number of potential solutions exist in the literature. For example, by imposing minimal flavor violation [32] on eq. (2.30), the $\mathcal{M}^{(2)}$ and $\overline{\mathcal{M}}^{(2)}$ can have masses as small as a few TeV. Or, as we discuss in section 3 , a discrete symmetry can be imposed (even if broken at $M_{\mathrm{P}}$ ) to forbid the $y_{u, d, e}^{\prime}$ couplings for all of the mesons except for $H_{u}$ and $H_{d}$.

\subsubsection{Color-triplet mesons}

As illustrated in eq. (2.29), we expect that gravitational effects induce electroweak scale $\mathcal{O}\left(\frac{\Lambda_{L} \Lambda_{R}}{M_{\mathrm{P}}}\right)$ supersymmetric masses for each of the five pairs of $\overline{\mathcal{M}}^{(3)} \mathcal{M}^{(3)}$ color triplets. Generically, color triplets with weak scale masses are very tightly constrained, especially because the interactions

$$
W_{\mathrm{bad}} \sim Q_{L} \overline{\mathcal{M}}^{(3)} L+\bar{u}_{R} \mathcal{M}^{(3)} \bar{e}_{R}+\bar{d}_{R} \mathcal{M}^{(3)}+\overline{\mathcal{M}}^{(3)} \bar{u}_{R} \overline{\mathcal{M}}^{(3)}+\ldots
$$

if present, would mediate fast proton decay. Fortunately, every term in eq. (2.31) is forbidden upon gauging $\mathrm{U}(1)_{X}=\mathrm{U}(1)_{B-L}$. Thus, $\mathcal{M}^{(3)}$ and $\overline{\mathcal{M}}^{(3)}$ are distinct from the Higgs color triplets which typically appear in $\mathrm{SU}(5)$ grand unified theories. In section 3 we explore the possibility that they could (along with the extra $\mathrm{SU}(2)_{L}$ doublets) serve as messengers for gauge-mediated supersymmetry breaking.

\subsection{Alternatives to $B-L$}

In addition to $B-L$, there are a number of other acceptable anomaly-free $\mathrm{U}(1)_{X}$ charge assignments for the Standard Model matter. While none are as attractive as $B-L$, in this section we sketch three alternatives: a " $5 /-3 / 1$ " pattern of $\mathrm{U}(1)_{X}$ charges within each generation; every matter superfield neutral under $\mathrm{U}(1)_{X}$; and an $L_{i}-L_{j}$ model. 


\begin{tabular}{|c|c|ccc|c|c|c|}
\hline & $\mathrm{SU}(5)_{1}$ & $S U_{3}$ & $S U_{2}$ & $\mathrm{U}(1)_{Y}$ & $\mathrm{SU}(5)_{2}$ & $\mathrm{U}(1)_{X}$ & $\mathrm{U}(1)_{\mathrm{PQ}}$ \\
\hline$\overline{\mathcal{M}}^{(3)}$ & $\mathbf{5}$ & $\overline{\mathbf{3}}$ & & $1 / 3$ & & 0 & 0 \\
$\overline{\mathcal{M}}^{(2)}$ & $\mathbf{5}$ & & $\mathbf{2}$ & $-1 / 2$ & & 0 & 0 \\
\hline $\mathcal{M}^{(3)}$ & & $\mathbf{3}$ & & $-1 / 3$ & $\overline{\mathbf{5}}$ & 0 & 0 \\
$\mathcal{M}^{(2)}$ & & & $\mathbf{2}$ & $1 / 2$ & $\overline{\mathbf{5}}$ & 0 & 0 \\
\hline $\mathcal{B}_{1}, \overline{\mathcal{B}}_{2}$ & & & & 0 & & 5 & $\pm 1+\alpha$ \\
$\overline{\mathcal{B}}_{1}, \mathcal{B}_{2}$ & & & & 0 & & -5 & $\pm 1-\alpha$ \\
\hline$Q_{L}$ & & $\mathbf{3}$ & $\mathbf{2}$ & $1 / 6$ & & $+q$ & 0 \\
$\bar{u}_{R}$ & & $\overline{3}$ & & $-2 / 3$ & & $+q$ & 0 \\
$\bar{d}_{R}$ & & $\overline{3}$ & & $1 / 3$ & & $-3 q$ & 0 \\
\hline$L$ & & & $\mathbf{2}$ & $-1 / 2$ & & $-3 q$ & 0 \\
$\bar{e}_{R}$ & & & & +1 & & $+q$ & 0 \\
$\bar{\nu}_{R}$ & & & & 0 & & $5 q$ & 0 \\
\hline$H_{u}$ & & & $\mathbf{2}$ & $1 / 2$ & & $-2 q$ & 0 \\
$H_{d}$ & & & $\mathbf{2}$ & $-1 / 2$ & & $2 q$ & 0 \\
\hline
\end{tabular}

Table 3. Charges of the matter fundamental superfields and Higgs doublets and composite baryons and mesons in the " $5 /-3 / 1 " \mathrm{U}(1)_{X}$ model.

\subsubsection{5/-3/1 model}

An alternative charge assignment is shown in table 3: $Q_{L}, \bar{u}_{R}$ and $\bar{e}_{R}$ fields have $\mathrm{U}(1)_{X}$ charge $q ; L$ and $\bar{d}_{R}$ have charge $-3 q$; and the $\bar{\nu}_{R}$ has charge $5 q$ to cancel the $\mathrm{U}(1)_{X}^{3}$. anomaly. Forbidding all $\mathrm{U}(1)_{\mathrm{PQ}}$-violating operators of dimension less than 10 requires:

$$
q \neq \pm 1, \pm \frac{1}{2}, \pm \frac{1}{3}, \pm \frac{1}{4}, \pm \frac{5}{2}, \pm \frac{5}{3},
$$

but otherwise $q$ is a free parameter describing a family of models. With this charge assignment the undesirable baryon and lepton number violating operators $L H_{u}, L L \bar{e}_{R}, Q L \bar{d}_{R}$ and $\bar{u}_{R} \bar{d}_{R} \bar{d}_{R}$ are all forbidden, and proton decay occurs via the dimension 5 operator $W \sim \bar{u}_{R} \bar{u}_{R} \bar{d}_{R} \bar{e}_{R} / M_{\mathrm{P}}$.

Unlike in the $B-L$ model, $\mathrm{U}(1)_{X}$ forbids the mesons $\mathcal{M}^{(2)}$ and $\overline{\mathcal{M}}^{(2)}$ from having Yukawa interactions with MSSM matter unless $q=0$. Thus, additional fundamental Higgs doublets $H_{u}+H_{d}$ with $\mathrm{U}(1)_{X}$ charges $\pm 2 q$ must be added to generate quark and lepton masses,

$$
W_{H}=\mu H_{u} H_{d}+y_{u} Q_{L} H_{u} \bar{u}_{R}+y_{d} Q_{L} H_{d} \bar{d}_{R}+y_{e} L H_{d} \bar{e}_{R}+y_{\nu} L H_{u} \bar{\nu}_{R} .
$$

As in the MSSM with fundamental Higgs doublets, there is no a priori reason for $\mu$ to be at the weak scale.

Renormalizable couplings between the mesons $\mathcal{M}$ and $\overline{\mathcal{M}}$ and the MSSM fields are mediated exclusively by gauge interactions. Direct couplings in the superpotential are suppressed, beginning with the dimension-7 operators $(\mathcal{M} \overline{\mathcal{M}}) H_{u} H_{d}$. Direct couplings which 
would allow the mesons to decay entirely into the Standard Model depend sensitively on $q$, with the operators permitting prompt decay also typically violating $\mathrm{U}(1)_{\mathrm{PQ}}$ and forbidden by eq. (2.32). As consequence, the lightest mesons tend to have long lifetimes, and for some values of $q$ can be absolutely stable and bounded by the strong constraints on colored or charged cosmological relic particles.

\subsection{2 $\mathrm{q}=0$ : neutral MSSM}

In the limit $q \rightarrow 0$, the MSSM decouples from $\mathrm{U}(1)_{X}$. This assignment allows for Yukawa interactions between the mesons and MSSM matter, permitting $\mathcal{M}^{(2)}$ and $\overline{\mathcal{M}}^{(2)}$ to play the role of the MSSM Higgs doublets, with $\mathcal{O}\left(\Lambda_{L} \Lambda_{R} / M_{\mathrm{P}}\right)$ supersymmetric masses as in eq. (2.29). However, $\mathrm{U}(1)_{X}$ no longer forbids the problematic operators of eq. (2.31) or

$$
W_{\mathrm{bad}}^{\prime} \sim L \mathcal{M}^{(2)}+L L \bar{e}_{R}+Q L \bar{d}_{R}+\bar{u}_{R} \bar{d}_{R} \bar{d}_{R}
$$

Among the potentially disastrous consequences of $W_{\mathrm{bad}}^{\prime}$ is a short proton lifetime. This problem is averted in the MSSM by imposing a $\mathbb{Z}_{2} R$ parity, which ensures that the superpotential respects the $B-L$ global symmetry. Upon imposing $R$ parity or some other discrete symmetry on the $q=0$ model, the superpotential comes to resemble that of the $B-L$ axion model in all respects except one: if $q=0$ the right-handed neutrino is a singlet under the gauge symmetries, at which point it can be safely omitted.

\subsection{3 $L_{i}-L_{j}$ models}

The Standard Model also admits anomaly-free U(1) symmetries for which charges are not uniform across all three generations. The combinations of $L_{\mu}-L_{\tau}$ and $L_{e}-L_{\tau}$ are among the phenomenologically interesting alternatives. Models of this type are typically consistent with a composite $H_{u}$ and $H_{d}$, but as in the MSSM, an $R$ parity must be imposed on such models to ensure that all of the $B$ and $L$ violating operators of eq. (2.34) are forbidden.

\section{Gauge-mediated supersymmetry breaking}

Beyond the usual MSSM superfields, there are relatively few additional light degrees of freedom:

- The four baryons $\overline{\mathcal{B}}_{1,2}$ and $\mathcal{B}_{1,2}$ contain at most two light fields in the $\left\langle\mathcal{B}_{i}\right\rangle \neq 0$, $\left\langle\overline{\mathcal{B}}_{i}\right\rangle \neq 0$ vacuum. There is a chiral multiplet containing the composite axion.

- For $\mathrm{U}(1)_{X}$ gauge coupling $g_{X} \ll 1$, there is a $\mathrm{U}(1)_{X}$ vector supermultiplet with a mass $m_{X} \sim g_{X} f_{X}$, where $f_{X}$ is typically $\sim f_{a}$.

- The mesons $\mathcal{M}$ and $\overline{\mathcal{M}}$ have $\mathcal{O}\left(\Lambda_{L} \Lambda_{R} / M_{\mathrm{P}}\right)$ vectorlike masses. In the $B-L$ model and its variants, the lightest such $\mathrm{SU}(2)_{L}$ doublets are identified as the MSSM $H_{u}$ and $H_{d}$ leaving four heavier $\mathcal{M}^{(2)}+\overline{\mathcal{M}}^{(2)}$ pairs, and five color triplets $\mathcal{M}^{(3)}+\overline{\mathcal{M}}^{(3)}$.

In this section we explore how these mesons may be utilized as messengers of supersymmetry breaking. 
We parameterize the supersymmetry-breaking in a secluded sector as a set of one or more chiral superfields $X_{i}$ acquiring $F$-term expectation values,

$$
\langle X\rangle=\mathcal{X}+\theta^{2} \mathcal{F}_{X},
$$

with $\mathcal{F}_{X} \neq 0$. Introducing superpotential terms of the form $W \sim X \overline{\mathcal{M}}^{(3,2)} \mathcal{M}^{(3,2)}$ communicates supersymmetry breaking to the MSSM $[33,34]$. In the UV theory this superpotential originates from dimension-5 operators $\left(\bar{Q}_{2} \bar{Q}_{1}\right) X\left(Q_{1} Q_{2}\right) / M_{S}^{2}$, reducing in the IR to

$$
W_{s}=\lambda_{3}^{\prime i j}\left(\frac{\Lambda_{L} \Lambda_{R}}{M_{S}^{2}}\right) X \overline{\mathcal{M}}_{i}^{(3)} \mathcal{M}_{j}^{(3)}+\lambda_{2}^{\prime i j}\left(\frac{\Lambda_{L} \Lambda_{R}}{M_{S}^{2}}\right) X \overline{\mathcal{M}}_{i}^{(2)} \mathcal{M}_{j}^{(2)}
$$

where the indices $i, j=1 \ldots 5$, for some scale $M_{S} \gtrsim \sqrt{\Lambda_{L} \Lambda_{R}}$ which we take to be small compared to $M_{\mathrm{P}}$. It is convenient to absorb the factors of $\Lambda_{L} \Lambda_{R} / M_{S}^{2}$ into the definitions of $\lambda_{2,3}$ :

$$
\lambda_{2,3}^{i j}=\frac{\Lambda_{L} \Lambda_{R}}{M_{S}^{2}} \lambda_{2,3}^{\prime i j}
$$

As with the Yukawa couplings of eq. (2.30), the superpotential $W_{s}$ respects a global $\mathrm{U}(1)_{R}$ symmetry under which the mesons $\mathcal{M}$ and $\overline{\mathcal{M}}$ are neutral, and $X$ has charge +2 .

As discussed in section 2.2, Yukawa-like couplings between the matter fields and the four heavy $\mathcal{M}^{(2)}+\overline{\mathcal{M}}^{(2)}$ may introduce unacceptable flavor-changing neutral currents. A standard solution is to impose a "messenger parity" on the model, under which the Higgs $H_{u, d}$ are even, and the messengers $\mathcal{M}^{(2,3)}$ and $\overline{\mathcal{M}}^{(2,3)}$ are odd. Thus, the direct couplings between messenger $\mathrm{SU}(2)_{L}$ doublets and the matter fields are forbidden, and the problematic flavor-changing neutral currents are avoided. ${ }^{2}$ Imposing this $\mathbb{Z}_{2}$ symmetry reduces eq. (3.2) to:

$$
W_{s}=\lambda_{3}^{1,1} X \overline{\mathcal{M}}_{1}^{(3)} \mathcal{M}_{1}^{(3)}+\lambda_{2}^{1,1} X H_{d} H_{u}+\sum_{i=2 \ldots 5} \sum_{j=2 \ldots 5}\left(\lambda_{3}^{i j} X \overline{\mathcal{M}}_{i}^{(3)} \mathcal{M}_{j}^{(3)}+\lambda_{2}^{i j} X \overline{\mathcal{M}}_{i}^{(2)} \mathcal{M}_{j}^{(2)}\right) \text {, }
$$

where, if the messenger parity is derived from the global symmetries of the quarks $Q_{2}$ and $\bar{Q}_{2}$, we take the $\mathrm{SU}(3)_{c}$ triplets $\overline{\mathcal{M}}_{1}^{(3)}$ and $\mathcal{M}_{1}^{(3)}$ to be even under the $\mathbb{Z}_{2}$ messenger parity.

Since the mesons come in complete SU(5) multiplets, gauge unification at a scale $M_{\mathrm{GUT}}$ is preserved due to the fact that $\mathcal{M}^{(3)}+\mathcal{M}^{(2)}$ and $\overline{\mathcal{M}}^{(3)}+\overline{\mathcal{M}}^{(2)}$ form complete $\mathrm{SU}(5)_{\mathrm{SM}}$ multiplets. Following [35], the gauge coupling strength $\alpha_{\mathrm{GUT}}$ at the unification scale $M_{\mathrm{GUT}}$ is modified by

$$
\delta \alpha_{\mathrm{GUT}}^{-1}=-\frac{N_{f}}{2 \pi} \ln \frac{M_{\mathrm{GUT}}}{\mathcal{X}}
$$

where $N_{f}=N_{c}=5$. Requiring that $\mathrm{SU}(5)_{\mathrm{SM}}$ remains perturbative up to the unification scale imposes a lower bound on $\mathcal{X}$ :

$$
\mathcal{X} \gtrsim 10^{-13} \times M_{\mathrm{GUT}} \approx 2 \mathrm{TeV} .
$$

\footnotetext{
${ }^{2}$ The messenger parity is a discrete subgroup of the $\mathrm{SU}(5)_{1} \times \mathrm{SU}(5)_{2}$ flavor symmetry, and can be derived from the breaking pattern $\mathrm{SU}(5)_{1,2} \rightarrow \mathrm{SU}(4)_{1,2} \times \mathrm{U}(1)$ with $\mathbb{Z}_{2} \subset \mathbb{Z}_{4} \subset \mathrm{U}(1)$, where $H_{u, d}$ and the corresponding $\mathrm{SU}(3)_{c}$ triplets are invariant under the action of $\mathbb{Z}_{4}$.
} 
In addition to eq. (3.2), the meson messengers also acquire $\mathrm{U}(1)_{R}$ violating mass terms from the Planck scale effects, $\mu_{2,3} \sim \Lambda_{L} \Lambda_{R} / M_{\mathrm{P}}$, leading to a scalar mass matrix:

$$
\left(\mathcal{M}_{(2,3)}^{\dagger} \overline{\mathcal{M}}_{(2,3)}\right)\left(\begin{array}{cc}
\left(\lambda_{2,3} \mathcal{X}+\mu_{2,3}\right)^{\dagger}\left(\lambda_{2,3} \mathcal{X}+\mu_{2,3}\right) & \left(\lambda_{2,3} \mathcal{F}_{X}\right)^{\dagger} \\
\lambda_{2,3} \mathcal{F}_{X} & \left(\lambda_{2,3} \mathcal{X}+\mu_{2,3}\right)\left(\lambda_{2,3} \mathcal{X}+\mu_{2,3}\right)^{\dagger}
\end{array}\right)\left(\begin{array}{l}
\mathcal{M}_{(2,3)} \\
\overline{\mathcal{M}}_{(2,3)}^{\dagger}
\end{array}\right) .
$$

Performing $\mathrm{SU}(4)_{1,2} \times \mathrm{U}(1)_{1,2}$ rotations on the fields $\overline{\mathcal{M}}^{(2)}$ and $\mathcal{M}^{(2)}$, the matrices $\left(\lambda_{2} \mathcal{X}+\mu_{2}\right)$ and $\left(\lambda_{2} \mathcal{F}_{X}\right)$ can be simultaneously diagonalized and made real:

$$
M_{i}=\left(\lambda_{2} \mathcal{X}+\mu_{2}\right)_{i i}, \quad F_{i}=\left(\lambda_{2} \mathcal{F}_{X}\right)_{i i},
$$

with eigenvalues $M_{i}^{2} \pm F_{i}$. This basis also diagonalizes the scalar mass matrix of $\overline{\mathcal{M}}^{(3)}$ and $\mathcal{M}^{(3)}$ in the special case $\lambda_{2}=\lambda_{3}$ and $\mu_{2}=\mu_{3}$ (but not in general). Positivity of the (squared) messenger masses imposes a constraint on the $F$-term VEV of the superfield $X$ :

$$
\mathcal{F}_{X}<\frac{\mu_{2}^{2}}{\lambda_{2}}+2 \mu_{2} \mathcal{X}+\lambda_{2} \mathcal{X}^{2}
$$

for each pair of $\lambda_{2}^{i i}$ and $\mu_{2}^{i i}$ in the diagonal basis. Note that due to the compositeness of the messengers, the couplings $\lambda_{2,3}$ are suppressed by a factor $\Lambda_{L} \Lambda_{R} / M_{S}^{2}$ which may be much smaller than unity.

To produce the correct electroweak scale, the $M^{2}$ and $F$ terms for $H_{u}$ and $H_{d}$ must coincide. Taking $\lambda_{2}^{1,1} \sim \frac{\Lambda_{L} \Lambda_{R}}{M_{S}^{2}}$ and $\mu_{2}^{1,1} \sim \frac{\Lambda_{L} \Lambda_{R}}{M_{\mathrm{P}}}$, this condition implies a relationship between the scales $M_{S}, \mathcal{X}$ and $\mathcal{F}_{X}$ :

$$
\mathcal{F}_{X} \sim \Lambda_{L} \Lambda_{R}\left(\frac{\mathcal{X}}{M_{S}}+\frac{M_{S}}{M_{\mathrm{P}}}\right)^{2}
$$

Taking the simplifying case $\sqrt{\Lambda_{L} \Lambda_{R}} \sim f_{a} \sim 10^{11} \mathrm{GeV}$ and $M_{S} \gtrsim f_{a}$ in the limit $\mathcal{X}<10^{5} \mathrm{GeV}$, eq. (3.10) reduces to the condition $\sqrt{\mathcal{F}_{X}} \sim \frac{f_{a} M_{S}}{M_{\mathrm{P}}}$. An investigation of the extensions to the composite axion model satisfying this constraint would be an interesting opportunity for future work.

\section{Conclusions and outlook}

We explore a model with a composite axion in which an accidental Peccei-Quinn symmetry naturally emerges as a solution to the strong $\mathrm{CP}$ problem. Gravitational perturbations to the axion scalar potential are shown to be sufficiently suppressed in the $N_{c}=5$ model to permit an axion decay constant of $f_{a} \lesssim 3 \times 10^{11} \mathrm{GeV}$, even under the pessimistic assumptions that supersymmetry breaking induces the most dangerous $\mathrm{U}(1)_{\mathrm{PQ}}$-violating $A$-term potential, and that the higher-dimensional operators representing quantum gravitational effects are parameterized by $\mathcal{O}(1)$ coupling constants. In addition to providing a satisfactory solution to the axion quality problem, this composite framework is easily extended to any model of axion-like particles (ALPs) with masses much smaller than the scale of spontaneous symmetry breaking. 
The general $\mathrm{SU}(N)_{L} \times \mathrm{SU}(N)_{R} \times \mathrm{U}(1)_{X}$ axion model allows the Standard Model matter fields to carry nearly any anomaly-free $\mathrm{U}(1)_{X}$ charge assignment without negatively affecting the axion quality. In particular, attractive features emerge when $\mathrm{U}(1)_{X}$ is associated with gauging the Standard Model $B-L$ global symmetry. The leading terms in the superpotential are those of the MSSM, with none of the problematic $B$ or $L$ violating operators that would otherwise need to be forbidden by invoking a discrete "matter parity". Additionally, if the Higgs $H_{u}$ and $H_{d}$ are taken to be the lightest of the $\mathrm{SU}(2)_{L}$ charged mesons from $\mathrm{SU}(5)_{L}$ and $\mathrm{SU}(5)_{R}$ confinement, the dimension- 4 gravitationally-induced operator naturally generates an electroweak scale $\mu$ term for $f_{a} \sim 10^{11} \mathrm{GeV}$. Other choices of $\mathrm{U}(1)_{X}$ charge assignments share this feature, that the $\mathrm{SU}(2)_{L}$ charged mesons have the same quantum numbers as $H_{u}$ and $H_{d}$, and could therefore produce a composite Higgs with a TeV scale $\mu$ term.

The low energy phenomenology largely resembles the MSSM plus a chiral superfield containing the standard QCD axion, axino, and a saxion. More unique are the presence of meson fields in vectorlike color triplet and electroweak doublet representations. In theories in which the lightest weak doublet pair are identified as the MSSM Higgs superfields, they will have $\sim \mathrm{TeV}$ masses. Their detailed phenomenology depends on the $\mathrm{U}(1)_{X}$ charge assignments and some choices of (perhaps slightly broken) global symmetries, and their presence indicates that the Large Hadron Collider could potentially uncover clues to higher scale physics. Alternatively, some of these fields could play the role of messengers, leading to a picture in which supersymmetry-breaking is mediated by gauge interactions.

Among the many opportunities for future work, some promising directions include developing the supersymmetry breaking sector, explaining the pattern of Yukawa couplings in the MSSM, or exploring the cosmological implications of the composite model in the early universe.

\section{Acknowledgments}

The authors are grateful for conversations with Arvind Rajaraman, Nathaniel Craig and Csaba Csaki. This work is supported in part by NSF Grant No. PHY-1620638, and was performed in part at the Aspen Center for Physics, supported by the NSF grant PHY-1607611.

Open Access. This article is distributed under the terms of the Creative Commons Attribution License (CC-BY 4.0), which permits any use, distribution and reproduction in any medium, provided the original author(s) and source are credited.

\section{References}

[1] C.A. Baker et al., An improved experimental limit on the electric dipole moment of the neutron, Phys. Rev. Lett. 97 (2006) 131801 [hep-ex/0602020] [INSPIRE].

[2] J.M. Pendlebury et al., Revised experimental upper limit on the electric dipole moment of the neutron, Phys. Rev. D 92 (2015) 092003 [arXiv:1509.04411] [INSPIRE].

[3] R.D. Peccei and H.R. Quinn, Constraints imposed by CP conservation in the presence of instantons, Phys. Rev. D 16 (1977) 1791 [INSPIRE]. 
[4] M.A. Shifman, A.I. Vainshtein and V.I. Zakharov, Can confinement ensure natural CP invariance of strong interactions?, Nucl. Phys. B 166 (1980) 493 [INSPIRE].

[5] J.E. Kim and G. Carosi, Axions and the strong CP problem, Rev. Mod. Phys. 82 (2010) 557 [arXiv:0807.3125] [INSPIRE].

[6] P. Di Vecchia and G. Veneziano, Chiral dynamics in the large $N$ limit, Nucl. Phys. B 171 (1980) 253 [INSPIRE].

[7] J.E. Kim, Weak interaction singlet and strong CP invariance, Phys. Rev. Lett. 43 (1979) 103 [INSPIRE].

[8] A.R. Zhitnitsky, On possible suppression of the axion hadron interactions (in Russian), Sov. J. Nucl. Phys. 31 (1980) 260 [Yad. Fiz. 31 (1980) 497] [inSPIRE].

[9] M. Dine, W. Fischler and M. Srednicki, A simple solution to the strong CP problem with a harmless axion, Phys. Lett. B 104 (1981) 199 [INSPIRE].

[10] J. Preskill, M.B. Wise and F. Wilczek, Cosmology of the invisible axion, Phys. Lett. B 120 (1983) 127 [INSPIRE].

[11] L.F. Abbott and P. Sikivie, A cosmological bound on the invisible axion, Phys. Lett. B 120 (1983) 133 [INSPIRE].

[12] M. Dine and W. Fischler, The not so harmless axion, Phys. Lett. B 120 (1983) 137 [INSPIRE].

[13] S.B. Giddings and A. Strominger, Axion induced topology change in quantum gravity and string theory, Nucl. Phys. B 306 (1988) 890 [INSPIRE].

[14] M. Kamionkowski and J. March-Russell, Planck scale physics and the Peccei-Quinn mechanism, Phys. Lett. B 282 (1992) 137 [hep-th/9202003] [InSPIRE].

[15] S.M. Barr and D. Seckel, Planck scale corrections to axion models, Phys. Rev. D 46 (1992) 539 [INSPIRE].

[16] R. Kallosh, A.D. Linde, D.A. Linde and L. Susskind, Gravity and global symmetries, Phys. Rev. D 52 (1995) 912 [hep-th/9502069] [INSPIRE].

[17] L.F. Abbott and M.B. Wise, Wormholes and global symmetries, Nucl. Phys. B 325 (1989) 687 [INSPIRE].

[18] S.R. Coleman and K.-M. Lee, Wormholes made without massless matter fields, Nucl. Phys. B 329 (1990) 387 [INSPIRE].

[19] E.J. Chun and A. Lukas, Discrete gauge symmetries in axionic extensions of the SSM, Phys. Lett. B 297 (1992) 298 [hep-ph/9209208] [INSPIRE].

[20] K. Harigaya, M. Ibe, K. Schmitz and T.T. Yanagida, Peccei-Quinn symmetry from a gauged discrete R symmetry, Phys. Rev. D 88 (2013) 075022 [arXiv:1308.1227] [INSPIRE].

[21] K. Harigaya, M. Ibe, K. Schmitz and T.T. Yanagida, Peccei-Quinn symmetry from dynamical supersymmetry breaking, Phys. Rev. D 92 (2015) 075003 [arXiv: 1505.07388] [inSPIRE].

[22] L. Randall, Composite axion models and Planck scale physics, Phys. Lett. B 284 (1992) 77 [INSPIRE].

[23] L. Di Luzio, E. Nardi and L. Ubaldi, Accidental Peccei-Quinn symmetry protected to arbitrary order, Phys. Rev. Lett. 119 (2017) 011801 [arXiv: 1704.01122] [INSPIRE]. 
[24] B. Lillard and T.M.P. Tait, A composite axion from a supersymmetric product group, JHEP 11 (2017) 005 [arXiv: 1707.04261] [INSPIRE].

[25] H.-C. Cheng and D.E. Kaplan, Axions and a gauged Peccei-Quinn symmetry, hep-ph/0103346 [INSPIRE].

[26] H. Fukuda, M. Ibe, M. Suzuki and T.T. Yanagida, A "gauged" U(1) Peccei-Quinn symmetry, Phys. Lett. B 771 (2017) 327 [arXiv: 1703.01112] [InSPIRE].

[27] M. Duerr, K. Schmidt-Hoberg and J. Unwin, Protecting the axion with local baryon number, Phys. Lett. B 780 (2018) 553 [arXiv:1712.01841] [InSPIRE].

[28] N. Seiberg, Exact results on the space of vacua of four-dimensional SUSY gauge theories, Phys. Rev. D 49 (1994) 6857 [hep-th/9402044] [INSPIRE].

[29] K.A. Intriligator and N. Seiberg, Phases of $N=1$ supersymmetric gauge theories in four-dimensions, Nucl. Phys. B 431 (1994) 551 [hep-th/9408155] [INSPIRE].

[30] S. Chang and H. Georgi, Quantum modified mooses, Nucl. Phys. B 672 (2003) 101 [hep-th/0209038] [INSPIRE].

[31] A. Font, L.E. Ibáñez and F. Quevedo, Does proton stability imply the existence of an extra $Z^{0}$ ?, Phys. Lett. B 228 (1989) 79 [inSPIRE].

[32] M. Ciuchini, G. Degrassi, P. Gambino and G.F. Giudice, Next-to-leading QCD corrections to $B \rightarrow X_{s} \gamma$ in supersymmetry, Nucl. Phys. B 534 (1998) 3 [hep-ph/9806308] [INSPIRE].

[33] M. Dine, A.E. Nelson and Y. Shirman, Low-energy dynamical supersymmetry breaking simplified, Phys. Rev. D 51 (1995) 1362 [hep-ph/9408384] [INSPIRE].

[34] M. Dine, A.E. Nelson, Y. Nir and Y. Shirman, New tools for low-energy dynamical supersymmetry breaking, Phys. Rev. D 53 (1996) 2658 [hep-ph/9507378] [INSPIRE].

[35] G.F. Giudice and R. Rattazzi, Theories with gauge mediated supersymmetry breaking, Phys. Rept. 322 (1999) 419 [hep-ph/9801271] [INSPIRE]. 